\title{
Hydrogen Bonding in Alcohol Clusters: A Comparative Study by Infrared Cavity Ringdown Laser Absorption Spectroscopy
}

\author{
Robert A. Provencal, ${ }^{\dagger}$ Raphael N. Casaes, Katja Roth, Joshua B. Paul, $\$$ Chris N. Chapo, and \\ Richard J. Saykally* \\ Department of Chemistry, University of California, Berkeley, California 94720
}

\author{
Gregory S. Tschumper ${ }^{\S}$ and Henry F. Schaefer, III \\ Center for Computational Quantum Chemistry, University of Georgia, Athens, Georgia 30602-2525
}

Received: June 11, 1999; In Final Form: December 8, 1999

\begin{abstract}
Infrared cavity ringdown laser absorption spectroscopy has been used to study the $\mathrm{O}-\mathrm{H}$ stretching vibrations of jet-cooled ethanol and butanol clusters. Three bonded $\mathrm{O}-\mathrm{H}$ stretches were attributed to the ethanol dimer, indicating the presence of more than one conformer in the molecular beam. One unresolved feature was measured for both the trimer and tetramer. To facilitate spectral assignment, the vibrational frequencies and intensities have been calculated for the ethanol dimer using second-order Moller-Plesset perturbation theory. Three bonded $\mathrm{O}-\mathrm{H}$ stretches have also been measured for the butanol dimer, suggesting that more than one conformer is present in the molecular beam. Vibrational bands were also detected for the butanol trimer and tetramer.
\end{abstract}

\section{Introduction}

Hydrogen-bonded clusters have received much attention in the recent literature, largely owing to the concerted effort underway to fully characterize the behavior of condensed-phase water. Through the use of such diverse experimental techniques as terahertz, resonant ion-dip, and vibrational predissociation spectroscopies coupled with state of the art ab initio and dynamical calculations, the structural and dynamical properties of water clusters have been examined at unprecedented levels of detail. ${ }^{1,2}$ Central to this effort is the characterization of cooperative effects in the dynamics of hydrogen-bonded networks in water clusters. Alcohol complexes represent an important complimentary class of hydrogen-bonded systems, particularly owing to their role in solvation phenomena. In addition to structural and hydrogen-bonding changes caused by replacing a hydrogen atom with an alkyl group, alcohol clusters permit a characterization of the respective solvent-solvent interactions. A detailed investigation of the shift in $\mathrm{O}-\mathrm{H}$ vibrational frequency as a function of both cluster size and side chain length is a convenient probe of such effects.

While methanol clusters have received considerable attention in recent years, little information exists for longer chain alcohol clusters. Recently, Ehbrecht and Huisken investigated vibrational spectra of ethanol clusters using molecular beam depletion spectroscopy. ${ }^{3}$ Their study was limited to the spectral region between 870 and $1100 \mathrm{~cm}^{-1}$, thus investigating the symmetric and asymmetric $\mathrm{C}-\mathrm{C}-\mathrm{O}$ stretches and the in-plane and outof-plane bends. The complicated ethanol dimer spectrum was interpreted as evidence of multiple linear conformers, while a

† Present address: Biosciences and Biotechnology Group, Los Alamos National Laboratory, Los Alamos, NM 87545.

$\doteqdot$ Present address: Harvard University, Department of Chemistry and Chemical Biology, Cambridge, MA 02138.

$\S$ Present address: Laboratorium Fur Physicalische Chemie, Zurich, $\mathrm{CH}-$ 8092. less complicated trimer spectrum indicates a cyclic structure. Strong coupling of the vibrational motions as well as the fact that the ethanol monomer itself exists in both trans- and gaucheisomers affected their results. The crystal structure of solid ethanol has also been investigated. ${ }^{4}$ These studies have shown that the structure of crystalline ethanol consists of unit cells with four ethanol molecules each and exhibits infinite zigzag chains of hydrogen bonds. Sarkar and Joarder have also investigated the structure of liquid ethanol at room temperature by X-ray diffraction. ${ }^{5}$ Their analysis suggests that hexamer clusters are abundant under these conditions.

Although several theoretical studies of methanol clusters have appeared recently, ${ }^{6-8}$ very few theoretical calculations have been performed on ethanol complexes. Ethanol presents a more formidable computational problem owing to its larger size as well as the existence of two stable monomer conformers. In the trans-monomer, the torsional angle between the hydroxyl hydrogen and terminal carbon about the $\mathrm{CO}$ bond $\left(\tau_{\mathrm{HOCC}}\right)$ is exactly $180^{\circ}$. For the gauche-monomer, $\tau_{\text {HOCC }}$ lies near $\pm 60^{\circ}$. Though the trans-monomer is slightly more energetically stable, the gauche-monomer is favored statistically by a ratio of $2: 1$. Hoang et al. calculated the infrared spectrum of the ethanol monomer, ${ }^{9}$ in agreement with early experimental work of Perchard and Josien. ${ }^{10}$ More recently, Ehbrecht and Huisken ${ }^{3}$ determined the minimum-energy structures of small ethanol clusters using an experimentally derived monomer geometry and an intermolecular pair potential developed by Jorgensen. ${ }^{11}$ The existence of trans- and gauche-conformers gives rise to several different isomers for each cluster. For the trimer, the minimum-energy structure was determined to be a cyclic ring consisting exclusively of trans-monomers. The lowest-energy dimer structures were predicted to have a trans-monomer as the hydrogen bond. During the preparation of this article, a density functional theory (DFT) study of ethanol dimers and cyclic ethanol trimers was reported by Gonzales et al. ${ }^{12}$ Similar trends 
were reported for the relative stability of the dimers and trimers. The work of Gonzalez et al. is the most extensive study to date of ethanol clusters by electronic structure. ${ }^{12}$ Following the classification of Gonzales et al., gauche is abbreviated herein as ga and trans as tr. When specifying a specific $\left(\mathrm{CH}_{3} \mathrm{CH}_{2}-\right.$ $\mathrm{OH})_{2}$ conformer, the configuration of the hydrogen bond donor is listed first.

Infrared cavity ringdown laser absorption spectroscopy (IRCRLAS), ${ }^{13-15}$ pioneered by Scherer et al., has been shown to be a useful technique for studying structures and hydrogen bond rearrangement dynamics in hydrogen-bonded systems. ${ }^{15-17}$ Recently, we reported an IR-CRLAS study of hydrogen-bonded methanol clusters. ${ }^{8}$ Paul et al. have also used IR-CRLAS to study the $\mathrm{O}-\mathrm{D}$ stretching and $\mathrm{H}-\mathrm{O}-\mathrm{H}$ bending vibrations of gaseous water clusters for the first time. ${ }^{18,17}$ These results facilitated the quantitative determination of the water pair potential, recently reported by Fellers et al. ${ }^{2}$ Here, we report the results of an IR-CRLAS study of hydrogen-bonded ethanol and butanol complexes as well as the results of theoretical calculations on the ethanol dimer performed at the MP2 level of theory.

\section{Experimental Section}

The Berkeley IR-CRLAS spectrometer used to obtain the present results has been described in recent publications. ${ }^{15,17,19}$ Briefly, tunable infrared radiation was generated by Raman shifting a Nd:YAG (Continuum NY 82) pumped pulsed dye laser (Lambda Physik, FL3002e). The Raman shifter consists of a $3.5 \mathrm{~m}$ high-pressure multipass cell (Herriot configuration) containing 150 psi of $\mathrm{H}_{2}$. After 17 passes, the $20-25 \mathrm{~mJ} /$ pulse of fundamental radiation $(617-650 \mathrm{~nm})$ generated $0.2-0.5 \mathrm{~mJ} /$ pulse of infrared $(2.7-3.4 \mu \mathrm{m})$ in the third Stokes band with a line width of $0.2 \mathrm{~cm}^{-1}$. This tunable infrared radiation was then injected into the ringdown cavity consisting of a pair of highly reflective dielectric mirrors $(R \sim 0.9999)$. The light exiting the cavity through the second mirror was focused with a $10 \mathrm{~cm}$ lens onto a $0.5 \mathrm{~mm}$ diameter $\mathrm{LN}_{2}$-cooled $\mathrm{InSb}$ detector. This signal was amplified with a $1 \mathrm{MHz}$ bandwidth and then digitized, averaged (20 shots/wavelength), and fit by leastsquares to a first-order exponential decay. The cavity optical transit time divided by the measured decay time yields the perpass total cavity loss, which is recorded as a function of wavelength. Alcohol clusters are produced by bubbling helium carrier gas through an alcohol reservoir at room temperature and expanding the resultant mixture into a vacuum chamber through a 4 in. pulsed supersonic slit source. This procedure generally produces clusters with rotational temperatures near 5 K. ${ }^{17}$ A background pressure of $\sim 200$ mTorr was maintained during the $40 \mathrm{~Hz}$ operation of the supersonic beam source by a $2500 \mathrm{cfm}$ Roots pump. The data were wavelength-calibrated from atmospheric water monomer transitions measured simultaneously with the ethanol data, which provided an accuracy of $0.2 \mathrm{~cm}^{-1}$.

\section{Theoretical Methods}

Concurrently, a series of ab initio computations were carried out on the ethanol monomer and dimer for comparison with the experiments. Computational procedures employed in this investigation were identical to those used in our recent study of methanol clusters. ${ }^{8}$ Equilibrium geometries, harmonic vibrational frequencies, and infrared (IR) intensities were determined for these two species using second-order Møller-Plesset perturbation theory (MP2). ${ }^{20}$ All ab initio computations were carried out using the GAUSSIAN $94^{21}$ system of programs.
Elements of the density matrixes were converged to six decimal places while the root-mean-square of these elements was converged to eight decimal places. This resulted in total energies that were converged to at least $1 \times 10^{-7}$ hartree.

Two basis sets, denoted DZP+diff and TZ2P+diff, were employed. The first is the same double- $\zeta$ basis set with polarization functions and diffuse functions from our earlier study of methanol clusters. ${ }^{8}$ This basis set was constructed from the Huzinaga-Dunning ${ }^{22,23}$ set of contracted double- $\zeta$ Gaussian functions with the addition of one set of p-type polarization functions for each $\mathrm{H}$ atom with an orbital exponent of $\alpha_{\mathrm{p}}(\mathrm{H})=$ 0.75 , and one set of five d-type polarization functions for each $\mathrm{C}$ and $\mathrm{O}$ atom with orbital exponents $\alpha_{\mathrm{d}}(\mathrm{C})=0.75$ and $\alpha_{\mathrm{d}}(\mathrm{O})$ $=0.85$. To complete the $\mathrm{DZP}^{++}$basis set, one even-tempered s-type diffuse function was added to each $\mathrm{H}$ atom, $\alpha_{\mathrm{s}}(\mathrm{H})=$ 0.04415 , and a set of even-tempered s- and p-type diffuse functions was added to each $\mathrm{C}$ and $\mathrm{O}$ atom, $\alpha_{\mathrm{s}}(\mathrm{C})=0.04302$, $\alpha_{p}(C)=0.03629, \alpha_{s}(O)=0.08227$, and $\alpha_{p}(O)=0.06508 .{ }^{24}$ Similarly, the TZ2P+diff basis set consists of Dunning's triple- $\zeta$ contraction $^{23}$ of Huzinga's primitive Gaussian functions ${ }^{22}$ for $\mathrm{H}, \mathrm{C}$, and $\mathrm{O}$ augmented with two sets of polarization functions with orbital exponents $\alpha_{\mathrm{p}}(\mathrm{H})=1.5$ and $0.375, \alpha_{\mathrm{d}}(\mathrm{C})=1.5$ and 0.375 , and $\alpha_{d}(\mathrm{O})=1.7$ and 0.425. As for the DZP+dif basis, the TZ2P+dif basis is completed by adding of a set of even-tempered diffuse functions with orbital exponents $\alpha_{\mathrm{s}}(\mathrm{H})$ $=0.03016, \alpha_{s}(C)=0.04812, \alpha_{p}(C)=0.03389, \alpha_{s}(O)=$ 0.08993 , and $\alpha_{p}(\mathrm{O})=0.058$ 40. Thus for the DZP+dif basis there are a total of 93 spherical harmonic Gaussian basis functions. This number increases to 144 for the TZ2P+dif basis.

Beginning from the DFT-optimized dimer structures, ${ }^{12,25}$ equilibrium geometries, harmonic vibrational frequencies, and infrared (IR) intensities were determined using the available analytic MP2 gradients and second derivatives (second-order properties available only for the smaller DZP+diff basis). No symmetry constraints were placed upon any of the species studied, including the $C_{s}$ trans-monomer. Although several optimized geometrical parameters of the dimer appear to be identical to the precision reported, they were not constrained to be so. Residual Cartesian forces of the reported equilibrium structures were less than $5.0 \times 10^{-6}$ hartree/b. It is important to reiterate that the computed vibrational frequencies are harmonic in nature and the corresponding IR intensities were determined within the double-harmonic approximation. Owing to the floppy nature of $\left(\mathrm{CH}_{3} \mathrm{CH}_{2} \mathrm{OH}\right)_{2}$, care must be exercised when comparing theoretical and experimental values.

\section{Results}

I. Experimental Results. Ethanol. Typical IR-CRLAS spectra of gaseous ethanol clusters in the $\mathrm{O}-\mathrm{H}$ stretching region are shown in Figure 1. These were obtained at constant stagnation pressure while the ethanol concentration was varied using a needle valve. This method allows for maintenance of a relatively constant expansion temperature as the ethanol concentration is adjusted. ${ }^{17}$ Several distinct bands can be seen in the spectrum, as well as a large broad ethanol "ice" feature at large ethanol concentrations. By comparing the relative growth rates of the individual features as the ethanol concentration is increased, it is possible to determine the carrier of each band. On the basis of this procedure and the computed frequencies, we assign the three bands at 3531.2, 3539.5, and $3547.5 \mathrm{~cm}^{-1}$ to the ethanol dimer. The unresolved band at $3448.3 \mathrm{~cm}^{-1}$ can be assigned to the trimer, while the broad feature at $3267.6 \mathrm{~cm}^{-1}$ can be assigned to the tetramer. The intense feature that appears at large ethanol concentrations, shown in Figure 2, can be 

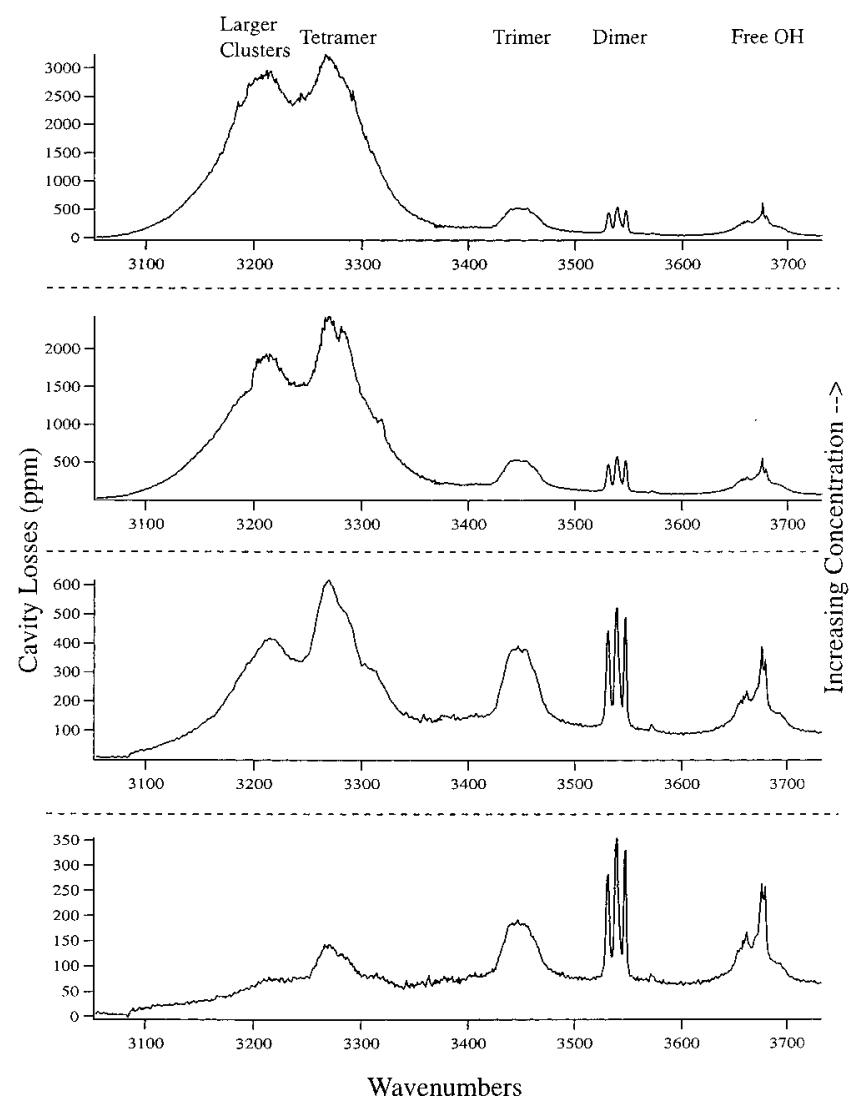

Figure 1. Typical IR-CRLAS spectra of the $\mathrm{O}-\mathrm{H}$ stretching region of gas-phase ethanol. Spectra are shown as a function of increasing ethanol concentration with constant source stagnation pressure.

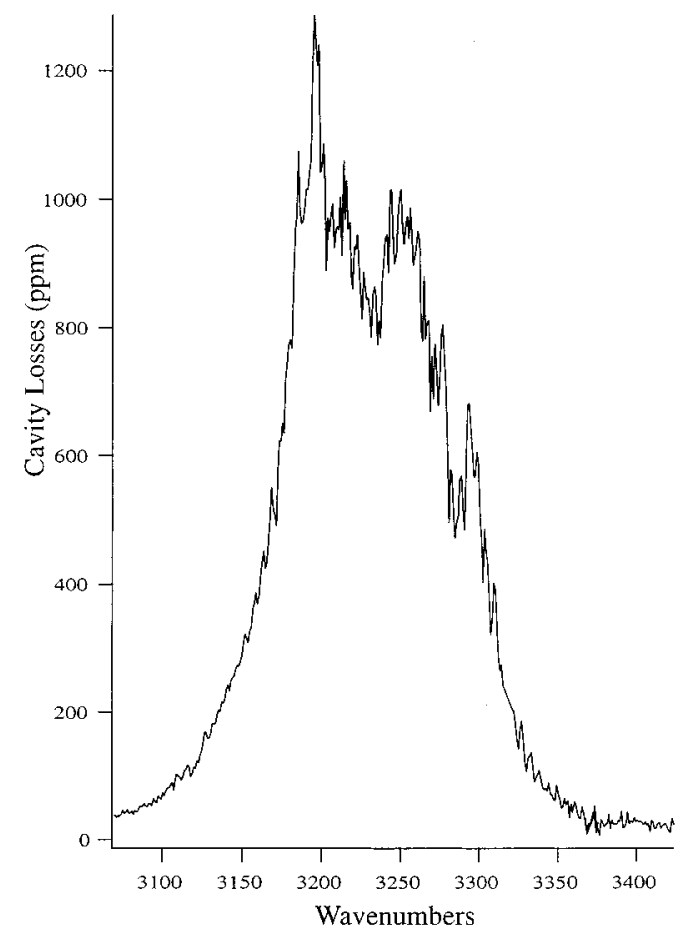

Figure 2. Broad background feature measured at high ethanol concentrations. The data shown here represents the difference of the two highest ethanol concentration scans shown in Figure 1.

assigned to larger clusters. As in previous studies of water and methanol, our source produces clusters that exhibit bulklike properties at high concentrations of ethanol.
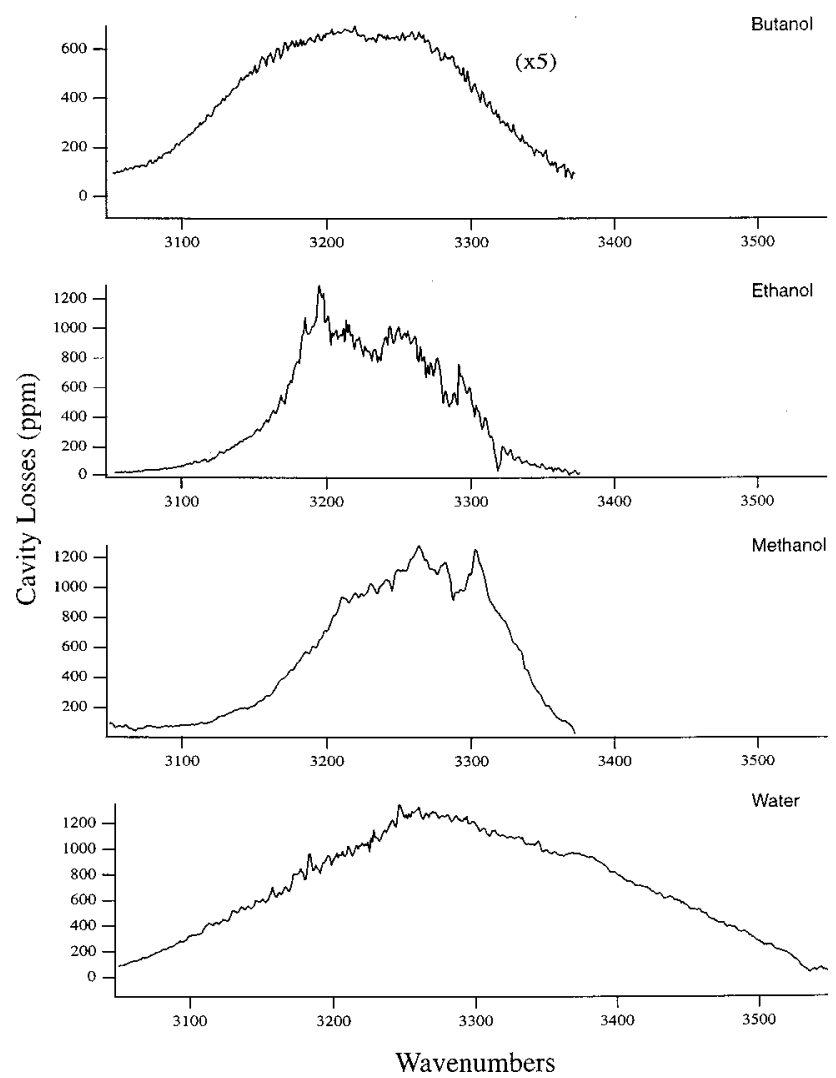

Figure 3. Comparison of the "icelike" feature measured for clusters of water, methanol, ethanol, and butanol. Spectra represent the difference between the two highest concentration scans. The butanol signal is shown multiplied by a factor of 5 . Note the increasing redshift as a function of alkyl group size. The water data is taken from ref 27 , while the methanol data is from ref 8 .

A detailed search was conducted in the free $\mathrm{O}-\mathrm{H}$ stretch region near 3680 for transitions assignable to cluster molecules. To distinguish such features, it is necessary to subtract the background monomer absorption from the signal present in the molecular beam. In our previous study of methanol, this method permitted the identification of a small dimer feature slightly blue-shifted from the $\mathrm{O}-\mathrm{H}$ of the methanol monomer. ${ }^{8}$ The success of this method relied on the fact that all of the methanol monomer absorption was seen in the background gas. With ethanol, however, a significant amount of ethanol monomer absorption is seen in the molecular beam. As a result, any weak features due to clusters are indistinguishable from the much larger monomer signal. Although the free $\mathrm{O}-\mathrm{H}$ stretch of the ethanol dimer is almost certainly present, it is not possible to determine whether it is intense enough to be detected by our experiment.

Butanol. The $\mathrm{O}-\mathrm{H}$ stretching spectrum of butanol clusters was also investigated. Typical IR-CRLAS spectra of gaseous butanol clusters are shown in Figure 4. The lower vapor pressure of butanol required removal of the needle valve to measure appreciable signal. As a result, these data were obtained at varying source backing pressures. The three bands at 3532.8, 3543.5 , and $3552.0 \mathrm{~cm}^{-1}$ can be assigned to the butanol dimer. While the following assignments are not definitive owing to the low observed intensities, the weak feature at $3452.7 \mathrm{~cm}^{-1}$ is most likely attributable to the butanol trimer while the broad features at 3279.0 and $3216.8 \mathrm{~cm}^{-1}$ are likely due to tetramer and larger clusters. Despite the bulky alkyl side chain of butanol, the hydrogen bond network is still strong enough to display features resembling the condensed phase. 

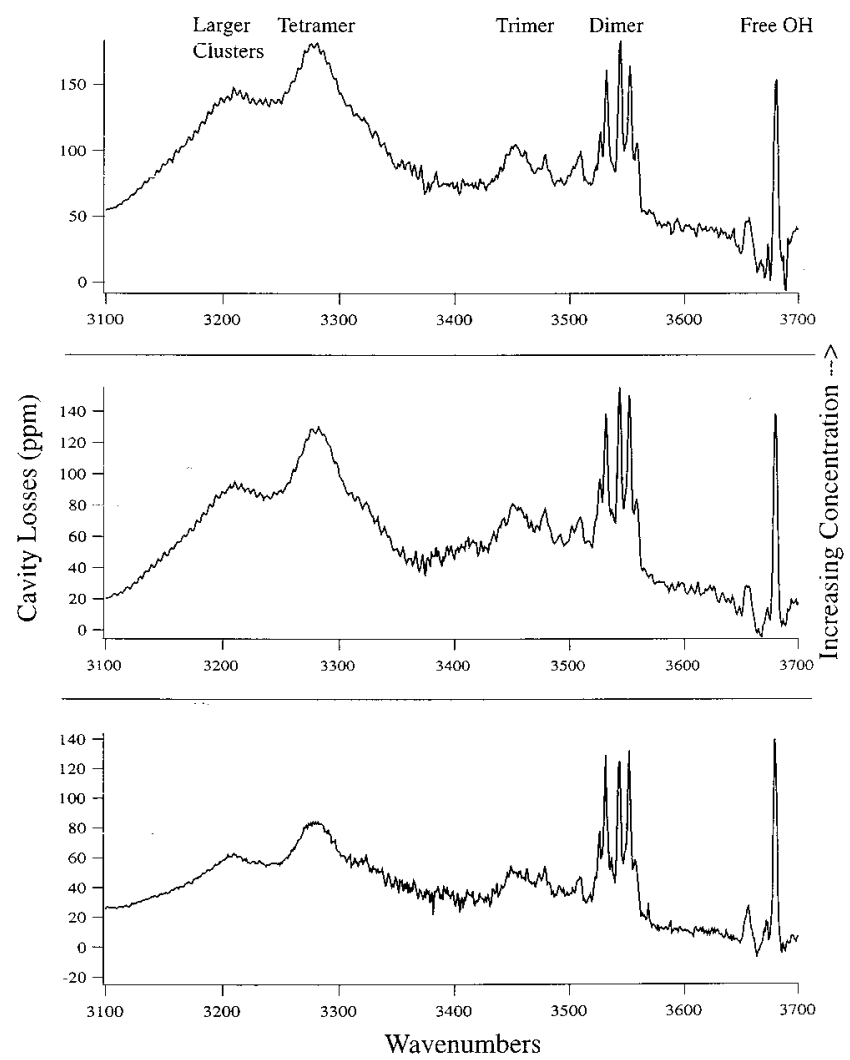

Figure 4. Typical IR-CRLAS spectra of the $\mathrm{O}-\mathrm{H}$ stretching region of gas-phase butanol. Spectra are shown as a function of increasing source backing pressure.

TABLE 1: Selected Equilibrium Properties of the Ethanol Gauche- and Trans-Monomers ${ }^{a}$

\begin{tabular}{|c|c|c|c|c|}
\hline $\begin{array}{l}\text { method } \\
\text { basis }\end{array}$ & $\begin{array}{c}\text { MP2 } \\
\text { DZP+rrrdif }\end{array}$ & $\begin{array}{c}\text { MP2 } \\
\text { TZ2P+dif }\end{array}$ & $\begin{array}{c}\text { B3LYP }^{b} \\
6-311+\mathrm{G}(\mathrm{d}, \mathrm{p})\end{array}$ & $\begin{array}{c}\text { B3LYP } b, c \\
6-311+\mathrm{G}(3 \mathrm{df}, 2 \mathrm{p})\end{array}$ \\
\hline \multicolumn{5}{|c|}{ Trans-Monomer } \\
\hline$E_{\text {TOTAL }}$ & -154.60134 & -154.69055 & -154.09506 & -155.10511 \\
\hline$E_{+\mathrm{ZPVE}}$ & -154.52006 & & -155.01549 & \\
\hline$r_{\mathrm{OH}}$ & 0.970 & 0.960 & 0.962 & \\
\hline$\tau_{\mathrm{HOCC}}$ & 180.0 & 180.0 & 180.0 & \\
\hline$\omega_{\mathrm{OH}}$ & 3874 & & 3842 & \\
\hline$I_{\mathrm{OH}}$ & 31 & & 28 & \\
\hline \multicolumn{5}{|c|}{ Gauche-Monomer } \\
\hline$E_{\text {TOTAL }}$ & -154.60128 & -154.69021 & -154.09496 & -155.10499 \\
\hline$E_{+\mathrm{ZPVE}}$ & -154.51973 & & -155.01528 & \\
\hline$r_{\mathrm{OH}}$ & 0.970 & 0.960 & 0.963 & \\
\hline$\tau_{\mathrm{HOCC}}$ & -58.0 & -60.8 & -60.8 & \\
\hline$\omega_{\mathrm{OH}}$ & 3863 & & 3827 & \\
\hline$I_{\mathrm{OH}}$ & 24 & & 22 & \\
\hline
\end{tabular}

${ }^{a}$ The total energies with and without an unscaled harmonic ZPVE correction $\left(E_{+\mathrm{ZPVE}}\right.$ and $E_{\mathrm{TOTAL}}$, respectively) are given in hartrees. Bond lengths $(r)$ are listed in $\AA$, angles $(\tau)$ in deg, vibrational frequencies $(\omega)$ in $\mathrm{cm}^{-1}$, and IR intensities $(I)$ in $\mathrm{km} / \mathrm{mol} .{ }^{b} \mathrm{~B} 3 \mathrm{LYP}$ results from reference 12. ${ }^{c}$ At B3LYP/6-311+G(d,p) optimized geometries.

II. Theoretical Results. Selected properties of the trans- and gauche-monomers are presented in Table 1 and those of the four dimers in Table 2. For comparison, DFT results are included where available. ${ }^{12,25}$ The usual hydrogen-bonding characteristics are present. The geometries of the monomers change very little upon clustering with the exception of the donor $\mathrm{OH}$ bond lengths, which increase by approximately $0.01 \AA$. The donor $\mathrm{OH}$ stretch harmonic vibrational frequencies are shifted downfield by roughly $150 \mathrm{~cm}^{-1}$, and the corresponding doubleharmonic IR intensities increase by more than an order of magnitude. As with the methanol dimer, ${ }^{8}$ the structure of the hydrogen bond in the ethanol dimers is remarkably similar to
TABLE 2: Selected Equilibrium Properties of the Ethanol Dimmers $^{a}$

\begin{tabular}{|c|c|c|c|c|}
\hline $\begin{array}{l}\text { method } \\
\text { basis }\end{array}$ & $\begin{array}{c}\mathrm{MP} 2 \\
\mathrm{DZP}+\text { dif }\end{array}$ & $\begin{array}{c}\text { MP2 } \\
\text { TZ2P+dif }\end{array}$ & $\begin{array}{c}\text { B3LYP } \\
6-311+\mathrm{G}(\mathrm{d}, \mathrm{p})\end{array}$ & $\begin{array}{c}\text { B3LYP }{ }^{b, c} \\
6-311+\mathrm{G}(3 \mathrm{df}, 2 \mathrm{p})\end{array}$ \\
\hline \multicolumn{5}{|c|}{ ga-tr Dimer } \\
\hline$E_{\text {TOTAL }}$ & -309.21563 & -309.39190 & -310.19882 & -310.21778 \\
\hline$E_{+\mathrm{ZPVE}}$ & -309.05048 & & -310.03751 & \\
\hline$r_{\mathrm{H}} \cdots \mathrm{O}$ & 1.881 & 1.892 & 1.922 & \\
\hline$r_{\mathrm{O}} \cdots \mathrm{O}$ & 2.854 & 2.857 & 2.889 & \\
\hline$\theta_{\mathrm{OH} \cdots \mathrm{O}}$ & 173.4 & 173.5 & 173.4 & \\
\hline $\mathrm{d}-r_{\mathrm{OH}}$ & 0.978 & 0.969 & 0.971 & \\
\hline $\mathrm{a}-r_{\mathrm{OH}}$ & 0.971 & 0.961 & 0.963 & \\
\hline $\mathrm{d}-\tau_{\mathrm{HOCC}}$ & -60.8 & -61.8 & -67.3 & \\
\hline $\mathrm{a}-\tau_{\mathrm{HOCC}}$ & 178.8 & 179.7 & 182.1 & \\
\hline $\mathrm{d}-\omega_{\mathrm{OH}}$ & 3710 & & 3673 & \\
\hline $\mathrm{a}-\omega_{\mathrm{OH}}$ & 3860 & & 3838 & \\
\hline $\mathrm{d}-I_{\mathrm{OH}}$ & 417 & & 464 & \\
\hline $\mathrm{a}-I_{\mathrm{OH}}$ & 42 & & 40 & \\
\hline \multicolumn{5}{|c|}{$\mathbf{t r}-\mathbf{t r}$ Dimer } \\
\hline$E_{\text {TOTAL }}$ & -309.21537 & -309.39182 & -310.19907 & -310.21778 \\
\hline$E_{+\mathrm{ZPVE}}$ & -309.05042 & & -310.03787 & \\
\hline$r_{\mathrm{H} \cdots \mathrm{O}}$ & 1.872 & 1.884 & 1.916 & \\
\hline$r_{\mathrm{O}} \cdots \mathrm{O}$ & 2.846 & 2.847 & 2.884 & \\
\hline$\theta_{\mathrm{OH} \cdots \mathrm{O}}$ & 173.0 & 172.4 & 175.9 & \\
\hline $\mathrm{d}-r_{\mathrm{OH}}$ & 0.978 & 0.969 & 0.970 & \\
\hline $\mathrm{a}-r_{\mathrm{OH}}$ & 0.971 & 0.961 & 0.962 & \\
\hline $\mathrm{d}-\tau_{\mathrm{HOCC}}$ & 176.7 & 177.2 & 178.4 & \\
\hline $\mathrm{a}-\tau_{\mathrm{HOCC}}$ & 179.4 & 180.7 & 181.6 & \\
\hline $\mathrm{d}-\omega_{\mathrm{OH}}$ & 3708 & & 3681 & \\
\hline $\mathrm{a}-\omega_{\mathrm{OH}}$ & 3862 & & 3839 & \\
\hline $\mathrm{d}-I_{\mathrm{OH}}$ & 513 & & 541 & \\
\hline $\mathrm{a}-I_{\mathrm{OH}}$ & 42 & & 41 & \\
\hline \multicolumn{5}{|c|}{ ga-ga Dimer } \\
\hline$E_{\text {TOTAL }}$ & -309.21454 & -309.39062 & -310.19871 & -310.21766 \\
\hline$E_{+\mathrm{ZPVE}}$ & -309.04953 & & -310.03738 & \\
\hline$r_{\mathrm{H}} \cdots \mathrm{O}$ & 1.877 & 1.888 & 1.918 & \\
\hline$r_{\mathrm{O}} \cdots_{\mathrm{O}}$ & 2.850 & 2.848 & 2.886 & \\
\hline$\theta_{\mathrm{OH}} \cdots \mathrm{O}$ & 172.6 & 170.8 & 175.1 & \\
\hline $\mathrm{d}-r_{\mathrm{OH}}$ & 0.978 & 0.968 & 0.971 & \\
\hline $\mathrm{a}-r_{\mathrm{OH}}$ & 0.972 & 0.961 & 0.963 & \\
\hline $\mathrm{d}-\tau_{\mathrm{HOCC}}$ & -61.3 & -63.4 & -66.3 & \\
\hline $\mathrm{a}-\tau_{\mathrm{HOCC}}$ & -58.4 & -62.9 & -61.2 & \\
\hline $\mathrm{d}-\tau_{\mathrm{OH}}$ & 3711 & & 3676 & \\
\hline $\mathrm{a}-\omega_{\mathrm{OH}}$ & 3851 & & 3824 & \\
\hline $\mathrm{d}-I_{\mathrm{OH}}$ & 494 & & 523 & \\
\hline $\mathrm{a}-I_{\mathrm{OH}}$ & 34 & & 33 & \\
\hline \multicolumn{5}{|c|}{ tr-ga Dimer } \\
\hline$E_{\text {TOTAL }}$ & -309.21432 & -309.39062 & -310.19896 & -310.21785 \\
\hline$E_{+\mathrm{ZPVE}}$ & -309.04952 & & -310.03779 & \\
\hline$r_{\mathrm{H}} \cdots \mathrm{O}$ & 1.869 & 1.880 & 1.911 & \\
\hline$r_{\mathrm{O}} \cdots \mathrm{O}$ & 2.842 & 2.842 & 2.879 & \\
\hline$\theta_{\mathrm{OH}} \cdots \mathrm{O}$ & 173.1 & 171.9 & 176.2 & \\
\hline $\mathrm{d}-r_{\mathrm{OH}}$ & 0.978 & 0.968 & 0.970 & \\
\hline $\mathrm{a}-r_{\mathrm{OH}}$ & 0.971 & 0.961 & 0.963 & \\
\hline $\mathrm{d}-\tau_{\mathrm{HOCC}}$ & 178.8 & 178.4 & 180.3 & \\
\hline $\mathrm{a}-\tau_{\mathrm{HOCC}}$ & -58.1 & -62.2 & -70.2 & \\
\hline $\mathrm{d}-\omega_{\mathrm{OH}}$ & 3710 & & 3682 & \\
\hline $\mathrm{a}-\omega_{\mathrm{OH}}$ & 3853 & & 3825 & \\
\hline $\mathrm{d}-I_{\mathrm{OH}}$ & 611 & & 614 & \\
\hline $\mathrm{a}-I_{\mathrm{OH}}$ & 33 & & 33 & \\
\hline
\end{tabular}

${ }^{a}$ The total energies with and without an unscaled harmonic ZPVE correction $\left(E_{+\mathrm{ZPVE}}\right.$ and $E_{\mathrm{TOTAL}}$, respectively) are given in hartrees. Bond lengths $(r)$ are listed in $\mathrm{A}$, angles $(\theta$ and $\tau)$ in deg, vibrational frequencies $(\omega)$ in $\mathrm{cm}^{-1}$, and IR intensities $(I)$ in $\mathrm{km} / \mathrm{mol}$. The prefixes a- and d- indicate whether a parameter is for the hydrogen bond acceptor or donor monomer, respectively. ${ }^{b} \mathrm{~B} 3 \mathrm{LYP}$ results from refs 12 . $^{c} \mathrm{At}$ B3LYP/6-311+G(d,p) optimized geometries.

that of the water dimer. Substitution of a methyl or ethyl group for one of the $\mathrm{H}$ atoms of the water molecules of $\left(\mathrm{H}_{2} \mathrm{O}\right)_{2}$ does not significantly affect the configuration of the hydrogen bond. Another similarity between the methanol and ethanol dimers is that, other than the elongation of the donor $\mathrm{OH}$ bond already mentioned, the geometry of the monomers changes very little upon complexation. 
TABLE 3: Relative $\left(\Delta E_{\text {Total }}\right.$ and $\left.\Delta E_{+Z P V E}\right)$ and Dissociation Energies $\left(D_{\mathrm{e}}\right.$ and $\left.D_{0}^{\mathrm{h}}\right)$ of the Ethanol Monomers and Dimers ${ }^{a}$

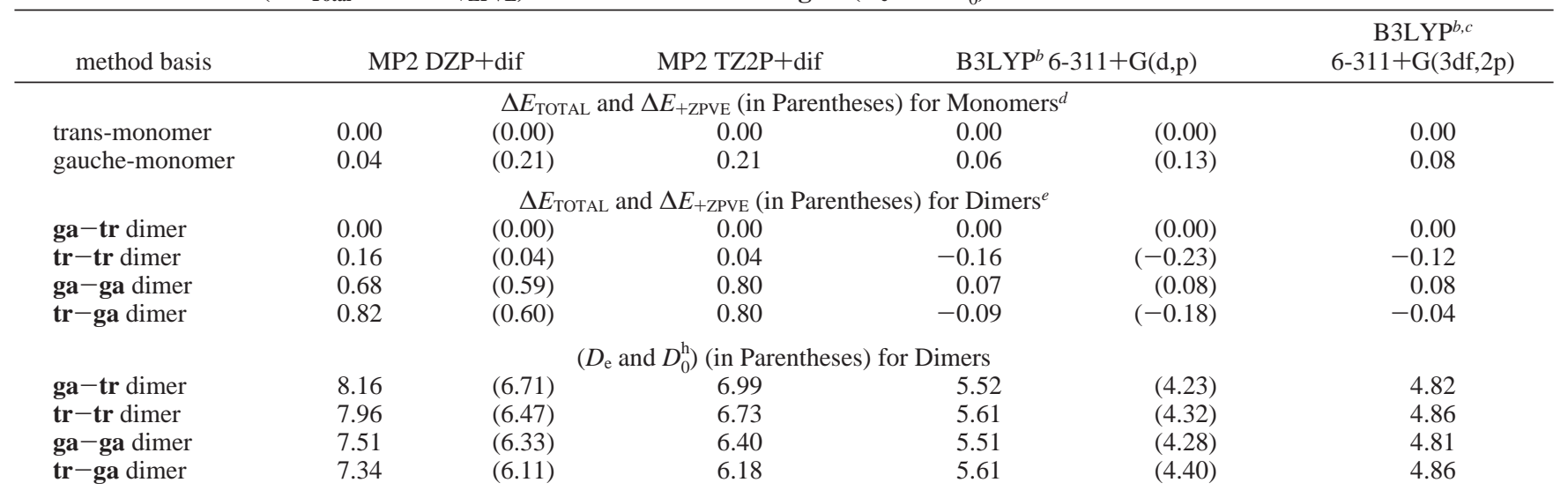

${ }^{a}$ All values are in $\mathrm{kcal} / \mathrm{mol}$. Dissociation energies $\left(D_{\mathrm{e}}\right.$ and $\left.D_{0}^{\mathrm{h}}\right)$ are computed from the energy difference of the total energies $\left(E_{\mathrm{TOTAL}}\right.$ and $\left.E_{+\mathrm{ZPVE}}\right)$ of the dimer and constituent monomers without correcting for BSSE. ${ }^{b}$ B3LYP results are from ref $12 .{ }^{c}$ At B3LYP/6-311+G(d,p) optimized geometry. ${ }^{d}$ Energy relative to the trans-monomer. ${ }^{e}$ Energy relative to the ga- $-\operatorname{tr}$ dimer.

The free $\mathrm{OH}$ stretch vibrational frequencies lie several wavenumbers away from the monomer transitions. However, in agreement with our experimental observations, it is unlikely that these modes would be detected since their double-harmonic IR intensities are at least an order of magnitude smaller than those for the bonded $\mathrm{OH}$ stretches. The hydrogen bond donor $\mathrm{OH}$ stretches of the four dimers are spaced very closely, separated by at most $4 \mathrm{~cm}^{-1}$ at the MP2/DZP+dif level. Given the typically broad nature of these red-shifted bands and the floppy nature of these clusters, assigning the experimental spectrum features to individual conformers from the ab initio data is not feasible.

The relative energies $\left(\Delta E_{\mathrm{TOTAL}}\right.$ and $\left.\Delta E_{+\mathrm{ZPVE}}\right)$ of the two monomers and four dimers are presented in Table 3. Although the trans-monomer is consistently the most stable of the monomers, the two conformers are energetically equivalent for all practical purposes. Unfortunately, the B3LYP and MP2 predictions for the dimers do not agree. With MP2, the ga- tr dimer is the most stable, and total energies increase in the following manner.

$$
E(\mathbf{g a}-\mathbf{t r}) \lesssim E(\mathbf{t r}-\mathbf{t r})<E(\mathbf{g a}-\mathbf{g a}) \lesssim E(\mathbf{t r}-\mathbf{g a})
$$

When using the B3LYP functional, the energies of the dimers are ordered quite differently.

$$
E(\mathbf{t r}-\mathbf{t r}) \lesssim E(\mathbf{t r}-\mathbf{g a})<E(\mathbf{g a}-\mathbf{t r}) \lesssim E(\mathbf{g a}-\mathbf{g a})
$$

While the DFT study suggests that a trans-monomer acting as the hydrogen bond donor produces the most stable dimer, MP2 computations suggest that a trans-monomer in the acceptor position is the key to stability. Given that the largest energy difference is well below $1 \mathrm{kcal} / \mathrm{mol}$, such a characterization is futile. The basis sets and electronic structure techniques used here and by Gonzales et al. ${ }^{12}$ cannot resolve such small energy differences. Only by systematically varying the basis sets and the level of electron correlation until convergence is reached with respect to both will definitive conclusions about the relative energies of the ethanol dimers be possible. Clearly this convergence has not been reached since, as one moves from the DZP+dif to the TZ2P+dif basis, the energy separation of the monomers increases from 0.04 to $0.21 \mathrm{kcal} / \mathrm{mol}$. Such a change is not observed with the B3LYP functional. However, the DFT monomer geometries were not optimized with the larger basis set. In light of the present MP2 and previous B3LYP results, one can only conclude that all four dimer conformers are energetically competitive.

Also included in Table 3 are the dissociation energies $\left(D_{\mathrm{e}}\right.$ and $D_{0}^{\mathrm{h}}$ ) of the dimers. These values have not been corrected for basis set superposition error. The general trends observed for relative energies also hold for the dissociation energies with only minor deviations for nearly degenerate isomers. For example, the most stable dimer also has the largest binding energy at a given level of theory. However, other standard correlations, such as between the binding energy and the hydrogen bond length or downfield shift of the $\mathrm{OH}$ stretch frequency, do not hold for the four ethanol dimer conformers at the theoretical levels presented here.

\section{Discussion}

This work represents the first study of the $\mathrm{O}-\mathrm{H}$ stretching spectrum of gaseous ethanol and butanol clusters. For the ethanol dimer, bands at 3531.2, 3539.5, and $3547.5 \mathrm{~cm}^{-1}$ were measured. Assignment of the ethanol dimer(s) as the carrier(s) of these bands is supported by both MP2 and B3LYP scaled harmonic vibrational frequencies. When scaling factors considered appropriate for the combinations of method and basis set (0.95 for MP2 and 0.96 for B3LYP) are employed, the redshifted $\mathrm{OH}$ stretch frequencies are predicted to lie in the range from 3523 to $3526 \mathrm{~cm}^{-1}$ at the MP2/DZP+dif level and from 3526 to $3535 \mathrm{~cm}^{-1}$ at the B3LYP/6-311+G(d,p).

The detection of three bands suggests that more than one dimer conformer is present in the molecular beam. This would not be surprising given the relative energetics of the dimer conformers discussed in section II. Although multiple bands can certainly arise from a single cyclic conformer, as now appears to be the case for methanol trimer, ${ }^{26}$ only one band should arise from a single dimer conformer. The band at 3539.5 $\mathrm{cm}^{-1}$ has a fwhm of $5.1 \mathrm{~cm}^{-1}$, while the other two bands, at 3532.8 and $3552.0 \mathrm{~cm}^{-1}$, have a fwhm of 4.1 and $3.8 \mathrm{~cm}^{-1}$, respectively. Furthermore, the $3539.5 \mathrm{~cm}^{-1}$ band's integrated intensity is approximately equal to the sum of the integrated intensities of the other two bands. This suggests that the band at $3539.5 \mathrm{~cm}^{-1}$ is a convolution of two bands. Unfortunately, owing to the low experimental resolution, attempts to fit the band at $3539.5 \mathrm{~cm}^{-1}$ to a convolution of Lorentzians could not be done. A higher resolution measurement of the three bands is needed before a detailed analysis of the band shapes can be done. Given the discrepancies between the theoretical predictions 
TABLE 4: Comparison of Selected Parameters of Water, Methanol, and Ethanol ga-tr Dimers Calculated at the MP2 TZ2P + dif Level of Theory ${ }^{a}$

\begin{tabular}{lccc}
\hline & \multicolumn{3}{c}{ cluster } \\
\cline { 2 - 4 } parameter & $\left(\mathrm{H}_{2} \mathrm{O}\right)_{2}$ & $\left(\mathrm{CH}_{3} \mathrm{OH}\right)_{2}$ & $\left(\mathrm{CH}_{3} \mathrm{CH}_{2} \mathrm{OH}\right)_{2}$ \\
\hline $\mathrm{d}-r_{\mathrm{O}-\mathrm{H}}$ & .960 & .967 & .969 \\
$\mathrm{a}-r_{\mathrm{O}-\mathrm{H}}$ & .966 & .960 & .961 \\
$r_{\mathrm{O} \cdots \cdot \mathrm{H}}$ & 1.949 & 1.884 & 1.892 \\
$r_{\mathrm{O}-\mathrm{O}}$ & 2.910 & 2.843 & 2.857 \\
$\theta_{\mathrm{O}-\mathrm{H}-\mathrm{O}}$ & 173.0 & 170.9 & 173.5 \\
$D_{\mathrm{e}}$ & 5.13 & 6.04 & 6.99
\end{tabular}

${ }^{a}$ Bond lengths are in $\AA$, angles in $\mathrm{deg}$, and energies in $\mathrm{kcal} / \mathrm{mol}$.

and the observed separation between the different bands, assignment of the spectral features to individual conformers is not possible. However the experimental results strongly suggest the presence of three or four conformers in the molecular beam. Our results also show that the band at $3448.3 \mathrm{~cm}^{-1}$ is the bonded $\mathrm{O}-\mathrm{H}$ stretch of the ethanol trimer. The relatively large frequency bandwidth of this feature (fwhm $\sim 21 \mathrm{~cm}^{-1} \mathrm{vs} \sim 11 \mathrm{~cm}^{-1}$ for $\left.\left(\mathrm{CH}_{3} \mathrm{OH}\right)_{3}\right)$ suggests that it is comprised of several unresolved trimer bands arising from a manifold of trimer conformers.

At large concentrations of ethanol, a broad red-shifted absorption centered near $3200 \mathrm{~cm}^{-1}$ appears (see Figure 2). Very similar features are also seen in our previous studies of water ${ }^{27}$ and methanol. ${ }^{8}$ As such, we attribute this feature to the presence of very large ethanol clusters produced in the molecular beam. Figure 3 compares these features measured for water, methanol, ethanol, and butanol. The "icelike" feature of butanol, shown multiplied by a factor of 5 in Figure 3, is clearly the weakest of the four molecules studied. Owing to its lower vapor pressure, it is fair to assume that this is due to the decreased number density of butanol in the molecular beam. The fact that we see features resembling the butanol tetramer suggests the formation of larger clusters despite the longer alkyl side chain.

Owing to the weak signal observed for the smaller butanol clusters, it was difficult to determine the changes in band intensity as a function of butanol concentration. As such, the presented band assignments are speculative. The three bands at $3532.8,3543.5$, and $3552.0 \mathrm{~cm}^{-1}$ can be assigned to the butanol dimer, owing in part to their similarity with the ethanol dimer peak positions. The small features appearing to the red of the butanol dimer are difficult to assign. The peak intensities are too small to accurately determine the changes in intensity as the butanol concentration is varied. We tentatively assign the broad feature at $3452 \mathrm{~cm}^{-1}$ to the butanol trimer, owing to its similarity to the ethanol trimer in both band position and band shape. The weak features at 3478 and $3507 \mathrm{~cm}^{-1}$ could be due to different conformers of the butanol trimer, but a definitive assignment is not possible. The stronger feature at $3279 \mathrm{~cm}^{-1}$ is assigned to the butanol tetramer owing to the similarity in the red-shifted band position with ethanol, methanol, and water.

There have been many theoretical studies comparing water, methanol, and ethanol clusters using different levels of theory. ${ }^{28}$ The results reported here, however, represent the first theoretical calculations of the ethanol monomer and dimer at the MP2 level of theory. As such, it is interesting to compare some of the important structural parameters of the dimers of water, methanol, and ga-tr ethanol, as shown in Table 4. All parameters were calculated at the MP2 level of theory with the TZ2P ++ basis set, including those of water. ${ }^{29}$ The first trend that should be noted is the increase in hydrogen bond dissociation energy as alkyl side chain length increases. The bond dissociation energies displayed in Table 4 are not corrected for basis set superposition errors, but this should not affect the overall trend in energies.

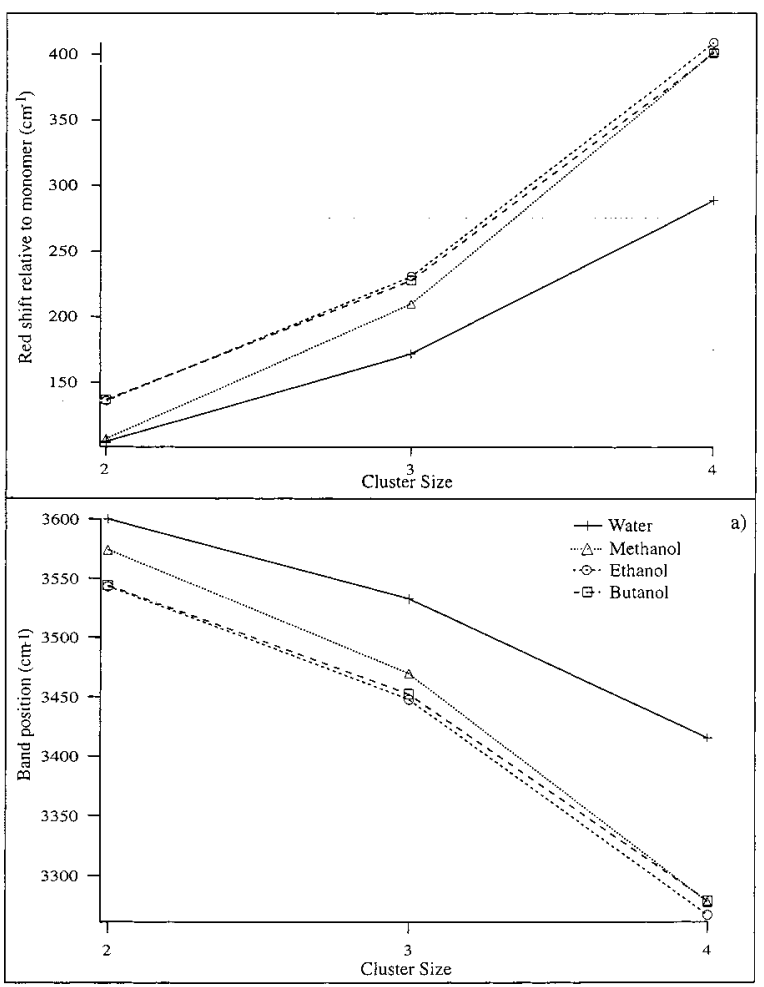

Figure 5. (a, bottom) Measured vibrational band positions vs cluster size for water, methanol, ethanol, and butanol. The increasing redshift of the band positions indicates a strengthening of the hydrogen bond. (b, top) Red-shift relative to the monomer $\mathrm{O}-\mathrm{H}$ stretch as a function of cluster size for a water, methanol, ethanol, and butanol. The red-shifts of water, methanol, and ethanol clusters follow the trend in the hydrogen bond strength (see Table 4). Butanol displays a redshift similar to ethanol's.

As the length of the side chain is increased, the $\mathrm{O}-\mathrm{H}$ bond length of the proton donor also lengthens. This is accompanied by a shortening of the $\mathrm{O}-\mathrm{O}$ bond distance of the methanol and ethanol dimers. This is a direct result of the increase in the individual hydrogen bond strength of the alcohol clusters. While the bond dissociation energy of the ethanol dimer is higher than that of the methanol dimer, the $\mathrm{O}-\mathrm{O}$ bond distance and the hydrogen bond length are slightly longer. This can be accounted for by the slightly more linear hydrogen bond of the ethanol dimer $\left(173.5^{\circ}\right.$ vs $\left.170.9^{\circ}\right)$.

The shift in $\mathrm{O}-\mathrm{H}$ frequency as a function of both cluster size and side chain length is a useful probe of the nature of the hydrogen bond network. Figure 5A displays the bonded $\mathrm{O}-\mathrm{H}$ band frequencies as a function of cluster size and side chain length. Figure 5B shows the shift of the band positions relative to the $\mathrm{O}-\mathrm{H}$ stretch of the corresponding monomers (water monomer stretching frequency is taken to be $\left.0.5\left(v_{1}+v_{2}\right)^{30,31}\right)$. Clearly evident is the well-known increasing red-shift as the cluster size increases, indicating a strengthening of the hydrogen bonding owing to cooperative interactions. The red-shift trend of the alcohol clusters compared to water clusters agrees very well with theoretical hydrogen bond dissociation energies (see Table 4). An exception is the methanol dimer, which has a redshift identical to that of the water dimer. A possible reason for this is the fact that the water dimer has a more linear hydrogen bond than the methanol dimer $\left(173.0^{\circ}\right.$ vs $\left.170.9^{\circ}\right)$. This effect disappears for the trimers, as the hydrogen bond angles are approximately equal $\left(151.1^{\circ}, 148.4^{\circ}, 151.4^{\circ}\right.$ for water ${ }^{32}$ vs $152.4^{\circ}, 151.2^{\circ}, 150.0^{\circ}$ for methanol $\left.{ }^{8}\right)$.

Ethanol displays a larger red-shift than methanol at all three measured cluster sizes, again in agreement with the calculated 
bond dissociation energies. Butanol's red-shift is nearly identical to ethanol's for the dimer and trimer but smaller for the tetramer, probably because of uncertainty in the butanol tetramer band position owing to the weak signal observed, broad nature of the band, and convolution with the "solidlike" feature. The band positions of the "solidlike" features displayed in Figure 3 were determined by fitting them to Gaussians. The water band position was found to be at $3292 \mathrm{~cm}^{-1}$, methanol at $3255 \mathrm{~cm}^{-1}$, ethanol at $3223 \mathrm{~cm}^{-1}$, and butanol at $3220 \mathrm{~cm}^{-1}$. Compared to water, the band position of the alcohols shift's to the red as the alkyl chain increases from a methyl to an ethyl group while increasing the side chain length from an ethyl to a butyl group produces no appreciable increase in red-shift. It should also be noted that the band shape measured for water is much broader that those measured for the alcohol clusters. This is undoubtedly due to the more complex hydrogen bonding present in large water clusters arising from double-donor hydrogen bonding. Current ab initio calculations predict that $\mathrm{O}-\mathrm{H}$ stretches of double-donor molecules occur in the region of $3400-3600 \mathrm{~cm}$ -1 , with a larger frequency spread relative to single donor stretches. ${ }^{33}$ This most likely explains the broader and more blueshifted water feature. These results indicate that an increase in size chain length from a methyl to an ethyl group produces a significant increase in hydrogen bond strength. However, increasing the side chain length from an ethyl to a butyl group appears to produce no significant strengthening of the hydrogen bonds.

\section{Summary}

Results of an IR-CRLAS study of ethanol and butanol clusters along with MP2 calculations of the four conformers of the ethanol dimer have been presented. Vibrational bands for clusters as large as the tetramer have been assigned for both alcohols. Observation of three ethanol dimer bands suggests the presence of at least three conformers in the molecular beam. The experimental and theoretical results suggest an increase in hydrogen bond strength as a function of side chain length up to ethanol. Increasing the side chain length from ethanol to butanol appears to have no significant impact on the hydrogen bond strength. It would be interesting to study the effect on hydrogen bond strength caused by replacing a primary alcohol with a secondary and tertiary alcohol.

Acknowledgment. The authors thank Dr. Arthur Suits for loaning the Raman shifter used in this work. This work is supported by the Air Force, Office of Sponsored Research (Grant F49620-96-1-0411) and the National Science Foundation (Grants CHE-9815397 and CHE-9727302).

\section{References and Notes}

(1) Liu, K.; Brown, M. G.; Cruzan, J. D.; Saykally, R. J. J. Phys. Chem. A 1997, 101, 9011-21.
(2) Fellers, R. S.; Braly, L. B.; Brown, M. G.; Leforestier, C.; Saykally, R. J. Science 1999, 284, 945 .

(3) Ehbrecht, M.; Huisken, F. J. Phys. Chem. A 1997, 101, 7768-77. 78.

(4) Anderson, A.; Benson, J.; Smith, W. Spectrosc. Lett. 1998, 31, 369-

(5) Sarkar, S.; Joarder, R. N. J. Chem. Phys. 1994, 100, 5118-22.

(6) Buck, U.; Ettischer, I. J. Chem. Phys. 1998, 108, 33-8. 601.

(7) Mo, O.; Yanez, M.; Elguero, J. J. Chem. Phys. 1997, 107, 3592-

(8) Provencal, R. A.; Paul, J. B.; Roth, K.; Chapo, C.; Casaes, R. N.; Saykally, R. J.; Tschumper, G. S.; Schaefer, H. F., III. J. Chem. Phys. 1999, $110,4258-67$. $6632-7$

(9) Hoang, D.; Lowe, M. A.; Alper, J. S. J. Phys. Chem. 1989, 93,

(10) Perchard, J. P.; Josien, M. L. J. Chem. Phys. 1968, 65, 1856.

(11) Jorgensen, W. L. J. Phys. Chem. 1986, 90, 1276.

(12) Gonzalez, L.; Mo, O.; Yanez, M. J. Chem. Phys. 1999, 111, 38553861.

(13) Scherer, J. J.; Voelkel, D.; Rakestraw, D. J.; Paul, J. B.; Collier, C. P.; Saykally, R. J.; O'Keefe, A. Chem. Phys. Lett. 1995, 245, 273-280.

(14) Paul, J. B.; Provencal, R. A.; Chapo, C.; Micheal, E.; Pettersson, A.; Saykally, R. J. Cavity Ringdown Spectroscopy; Oxford University Press: Washington, D.C., 1999; Vol. 720

(15) Paul, J. B.; Saykally, R. J. Anal. Chem. 1997, 69, 287-292.

(16) Paul, J. B.; Collier, C. P.; Saykally, R. J.; Scherer, J. J.; O’Keefe, A. J. Phys. Chem. A 1997, 101, 5211-14.

(17) Paul, J. B.; Provencal, R. A.; Chapo, C.; Pettersson, A.; Saykally, R. J. J. Chem. Phys. 1998, 109, 10201.

(18) Paul, J. B.; Provencal, R. A.; Chapo, C.; Roth, K.; Casaes, R. N.; Saykally, R. J. J. Phys. Chem. 1999, 103, 2972.

(19) Chapo, C. J.; Paul, J. B.; Provencal, R. A.; Saykally, R. J. J. Am. Chem. Soc. 1998, 120, 12956.

(20) Møller, C.; Plesset, M. S. Phys. Rev. 1934, 46, 618.

(21) Frisch, M. J.; Trucks, G. W.; Schlegel, H. B.; Gill, P. M. W. Johnson, B. G.; Robb, M. A.; Cheeseman, J. R.; Kieth, T.; Petersson, G. A.; Montgomery, J. A.; Raghavachari, K.; Al-Laham, M. A.; Zakrzewski, V. G.; Ortiz, J. V.; Foresman, J. B.; Cioslowski, J.; Stefanov, B. B.; Nanayakkara, M. C. A.; Peng, C. Y.; Ayala, P. Y.; Chen, W.; Wong, M. W.; Andres, J. L.; Replogle, E. S.; Gomperts, D. J. F. R.; Martin, R. L.; Binkley, J. S.; Defrees, D. J.; Baker, J.; Stewart, J. P.; Head-Gordon, M.; Gonzalez, C.; Pople, J. A. GAUSSIAN 94, Revision C.3; Gaussian, Inc. Pittsburgh, PA, 1995.

(22) Huzinaga, S. J. Chem. Phys. 1965, 42, 1293.

(23) Dunning, T. H. J. Chem. Phys. 1970, 53, 1784.

(24) Lee, T. J.; Schaefer, H. F. J. Chem. Phys. 1985, 83, 1784.

(25) Gonzalez, L.; Mo, O.; Yanez, M. Personal communication, 1999.

(26) Tschumper, G. S.; Gonzales, J. M.; Schaefer, H. F. J. Chem. Phys. 1999, 111, 3027-3034.

(27) Paul, J. B. Infrared Cavity Ringdown Laser Spectroscopy of Water Clusters. Ph.D., University of California, 1998.

(28) Scheiner, S. Hydrogen Bonding: A Theoretical Perspective, 1st ed.; Oxford University Press: Oxford, England, 1997.

(29) Tschumper, G. S.; Schaefer, H. F. Personal communication, 1999.

(30) Bleiber, A.; Sauer, J. Chem. Phys. Lett. 1995, 238, 243-52.

(31) Fröchtenicht, R.; Kaloudis, M.; Koch, M.; Huisken, F. J. Chem. Phys. 1996, 105, 6128-6139. 8792.

(32) Xantheas, S. S.; Dunning, T. H., Jr. J. Chem. Phys. 1993, 99, 8774-

(33) Knochenmuss, R.; Leutwyler, S. J. Chem. Phys. 1992, 96, 5233. 